\title{
Model study of the sign problem in a mean-field approximation
}

\section{Yoshimasa Hidaka}

RIKEN BNL Research Center, Brookhaven National Laboratory, Upton, New York 11973, USA

\begin{abstract}
We study the sign problem of the fermion determinant at nonzero baryon chemical potential. For this purpose we apply a simple model derived from Quantum Chromodynamics, in the limit of large chemical potential and mass. For SU(2) color, there is no sign problem and the mean-field approximation is similar to data from the lattice. For SU(3) color the sign problem is unavoidable, even in a mean-field approximation. We apply a phase-reweighting method, combined with the mean-field approximation, to estimate thermodynamic quantities. We also investigate the meanfield free energy using a saddle-point approximation [1].
\end{abstract}

The XXV International Symposium on Lattice Field Theory

July 30-4 August 2007

Regensburg, Germany 


\section{Introduction}

One of current thrusts of hadronic physics is to understand extreme conditions of temperature and/or density. The RHIC experiments reveal interesting features of a Quark-Gluon Plasma (QGP) phase above the critical temperature. In the core of a dense neutron star, various color superconductors should exist. Lattice calculations, based on Monte-Carlo simulation, is a powerful tool for the nonperturbative analysis of QCD. At zero baryon density, results from the lattice provide us with fundamental information, such as the phase transition temperature $T_{\mathrm{c}}$ [2], the equation of state [3], susceptibilities [4], the behavior of correlation functions, and so on.

On the other hand, at nonzero quark density lattice simulations have a serious sign problem: the quark determinant is complex in the presence of the baryon chemical potential, so Boltzmann weights are complex, and importantance sampling fails. There are ways to overcome the problem, including reweighting [5], Taylor expansion in the chemical potential [6], and analytical continuation from imaginary values of the chemical potential [7]. These methods are applicable when the chemical potential is small, and the temperature high. The sign problem, is even more intractable at low temperature and high density. In this work we study the sign problem in a toy model using a mean-field approximation.

Let us first see how the sign problem arises. The quark determinant is

$$
\operatorname{det} \mathscr{M}\left(\mu_{q}\right) \equiv \operatorname{det}\left[\gamma_{\mu} D^{\mu}+\gamma_{4} \mu_{q}+m_{q}\right]
$$

where $D^{\mu} \equiv \partial^{\mu}-i g A^{\mu}$ is the covariant derivative, $m_{q}$ is the quark mass and $\mu_{q}$ is the quark chemical potential. The quark determinant is complex except for $\mu_{q}=0$, due to a lack of gamma-five Hermiticity of $\mathscr{M}\left(\mu_{q}\right): \operatorname{det} \mathscr{M}\left(\mu_{q}\right)=\operatorname{det} \gamma_{5} \mathscr{M}\left(\mu_{q}\right) \gamma_{5}=\left\{\operatorname{det} \mathscr{M}\left(-\mu_{q}\right)\right\}^{*}$. In and of itself, a complex quark determinant is not necessarily fatal. While the quark determinant is complex for any given $A_{\mu}$, the functional integral over $A_{\mu}$ is real for real observables. This is seen from the relation

$$
\operatorname{det}\left[\gamma_{\mu}\left(\partial^{\mu}-i g\left(A^{\mu}\right)^{C}+\gamma_{4} \mu_{q}+m_{q}\right)\right]=\left\{\operatorname{det}\left[\gamma_{\mu} D^{\mu}+\gamma_{4} \mu_{q}+m_{q}\right]\right\}^{*}
$$

The real part of the quark determinant is $C$-even, and the imaginary part, $C$-odd. For a $C$-even $(C$ odd) observable, then, the imaginary (real) part of the determinant vanishes after integration over $A_{\mu}$. Accordingly the real problem is that the contribution of the quark determinant changes sign, depending upon $A_{\mu}$, and there is no known method to replace importance sampling.

\section{Model}

We analyze a simple model to see the sign problem in the mean-field approximation. The model is obtained by taking double limit of heavy mass, $m \rightarrow \infty$, and large chemical potential, $\mu \rightarrow \infty$, keeping the ratio $\varepsilon \equiv\left(e^{\mu_{q} a} / 2 m_{q} a\right)^{N_{\tau}}$ fixed. In the heavy quark limit all excited quarks are static, while antiquarks are suppressed at nonzero quark density, so that in the end, the quark determinant can be rewritten in terms of the Polyakov loop,

$$
e^{-S_{\mathrm{f}}[L]} \equiv \operatorname{det}\left[\gamma_{\mu} D^{\mu}+\gamma_{4} \mu_{q}+m_{q}\right] \rightarrow[\operatorname{det}(1+\varepsilon L)]^{N_{\mathrm{f}} / 4}
$$


where $L(\vec{x})=\prod_{\vec{x}_{4}} U_{\vec{x}_{4}}(\vec{x})$ is the Wilson line, which is a color matrix. Here we set $N_{\mathrm{f}}=4$ in order to avoid the rooting problem with staggered quarks. The determinant can be explicitly calculated:

$$
\operatorname{det} \mathscr{M}=\left\{\begin{array}{ll}
\prod_{\vec{x}}\left(1+\varepsilon^{2}+2 \varepsilon \ell\right) & \text { for } \mathrm{SU}(2) \\
\prod_{\vec{x}}\left(1+\varepsilon^{3}+3 \varepsilon \ell+3 \varepsilon^{2} \ell^{*}\right) & \text { for } \mathrm{SU}(3)
\end{array},\right.
$$

where the Polyakov loop is the trace of the Wilson line, $\ell(\vec{x})=\operatorname{tr} L(\vec{x}) / N_{\mathrm{c}}$ in the fundamental representation. One can easily check that $\mathrm{SU}(2)$ color does not have the sign problem since the Polyakov loop is always real, $-1 \leq \ell \leq 1$. For $\mathrm{SU}(3)$ case the Polyakov loop $\ell$ is complex valued, and the determinant is complex. We analyze these two cases in the next section.

For gluons, we take a simple action with nearest neighbor interactions between Polyakov loops,

$$
S_{\mathrm{g}}=-N_{\mathrm{c}}^{2} J \sum_{\mathrm{n} . \mathrm{n} .} \ell(\vec{x}) \ell^{*}(\vec{y})
$$

$J$ is a parameter which can be interpreted as the temperature of the system. In the strong coupling expansion, $J$ is related to the true temperture, $T$, through $J=\exp [-\sigma a / T]$, where $\sigma$ is the string tension. It is known that this action reproduces the gross features of the phase transition without quarks; i.e., a second-order phase transition for SU(2) color, and a first-order phase transition for $\mathrm{SU}(3)$ color. In this work we leave $J$ as a free parameter.

\section{Mean-field approximation}

At nonzero temperature the free energy is related to the functional integral as

$$
e^{-\beta V f}=Z(\beta, \mu)=\int D L \exp (-S)
$$

$\beta=1 / T$. Assuming that the action $S$ is real, $\exp (-S)$ is positive semidefinite, and in a mean-field approximation the free energy is:

$$
\begin{aligned}
e^{-\beta V f} & =\int D L \exp \left(-S_{\mathrm{mf}}(x)+S_{\mathrm{mf}}(x)-S\right)=Z_{\mathrm{mf}}(\beta)\left\langle\exp \left(S_{\mathrm{mf}}(x)-S\right)\right\rangle_{\mathrm{mf}} \\
& \geq Z_{\mathrm{mf}}(\beta) \exp \left(\left\langle S_{\mathrm{mf}}(x)-S\right\rangle_{\mathrm{mf}}\right) \equiv e^{-\beta V f(x)}
\end{aligned}
$$

The average $\langle\cdots\rangle_{\mathrm{mf}}$ is taken with respect to mean-field action $S_{\mathrm{mf}}(x) ; x$ is a parameter of meanfield theory. In eq. (3.2), the first line in is an identity, while the second line follows from Jensen's inequality, $\langle(\exp \mathscr{O})\rangle \geq \exp (\langle\mathscr{O}\rangle)$. The mean-field free energy is larger or equal than the exact free energy for any $x$. The inequality ensures that the $f(x)$ has a minimum at $x=x_{0}$,

$$
\left.\frac{\partial f(x)}{\partial x}\right|_{x=x_{0}}=0, \quad \text { and }\left.\quad \frac{\partial^{2} f(x)}{\partial x^{2}}\right|_{x=x_{0}} \geq 0 .
$$

Our ansatz for the mean-field action is

$$
S_{\mathrm{mf}}[L] \equiv-\frac{x}{2} \sum_{\vec{x}}\left[\ell(\vec{x})+\ell^{*}(\vec{x})\right] .
$$


Once $S_{\mathrm{mf}}[L]$ is known, and $x$ determined, the expectation value of any observable $\mathscr{O}[L]$ is given by integrating with repsect to the Wilson line, $L$, over the group measure, with the action of meanfield theory, $S_{\mathrm{mf}}[L]:\langle\mathscr{O}[L]\rangle \simeq\langle\mathscr{O}[L]\rangle_{\mathrm{mf}}$. If the action is not real, inequality is not ensured, and convexity is violated. This how the sign problem manifests itself in a mean-field approximation, and occurs for three or more colors. Charge-conjugation symmetry is violated at non-zero quark density, which is seen from $\langle\ell\rangle \neq\left\langle\ell^{*}\right\rangle$. Here we note that both $\langle\ell\rangle$ and $\left\langle\ell^{*}\right\rangle$ are real valued, from the argument in Sec1. This difference has been observed in both lattice simulations [6 and in other models $\llbracket 8]$. It is necessary to extend the mean-field ansatz (3.4) to include two variables, $x$ and $y$, in order to represent the difference between $\langle\ell\rangle$ and $\left\langle\ell^{*}\right\rangle$ :

$$
S_{\mathrm{mf}}=-\frac{x}{2} \sum_{\vec{x}}\left[\ell(\vec{x})+\ell^{*}(\vec{x})\right]-\frac{y}{2} \sum_{\vec{x}}\left[\ell(\vec{x})-\ell^{*}(\vec{x})\right] .
$$

While the mean-field action is complex, $x$ and $y$ are real, so that after integrating over $L$, the free energy $f_{\mathrm{mf}}(x, y)$ is a real function of $x$ and $y$. Their values are then determined by requiring that the free energy is a stationary point. At $\mu_{q} \neq 0, y \neq 0$. It turns out that about the stationary point, while the free energy $f_{\mathrm{mf}}(x, y)$ is minimal in the $x$ direction, it is maximal in the $y$ direction. That is, the solution is a saddle-point in $x$ and $y$, consistent with Ref. [8].

The phase reweighting method is one way to deal with the complexity of the action. The magnitude of the quark determinant is $C$-even, while its phase is $C$-odd. Accordingly, the quark action is

$$
S_{\mathrm{f}}=S_{\mathrm{f}}^{\mathrm{mag}}+i \Theta[L]
$$

where

$$
\begin{aligned}
& S_{\mathrm{f}}^{\mathrm{mag}}=-\sum_{\vec{x}} \ln \left|1+\varepsilon^{3}+3 \varepsilon \ell+3 \varepsilon \ell^{*}\right|, \\
& \Theta[L]=-\sum_{\vec{x}} \arg \left(1+\varepsilon^{3}+3 \varepsilon \ell+3 \varepsilon^{2} \ell^{*}\right) .
\end{aligned}
$$

With these definitions the expectation value of $\mathscr{O}[L]$ is

$$
\langle\mathscr{O}[L]\rangle \simeq\left\langle\mathscr{O}[L] e^{-i \Theta[L]}\right\rangle_{\mathrm{mf}} /\left\langle e^{-i \Theta[L]}\right\rangle_{\mathrm{mf}}
$$

Here $S_{\mathrm{mf}}[L]$ or $x$ is fixed from the free energy with the action $S_{\mathrm{g}}[L]+S_{\mathrm{f}}^{\mathrm{mag}}[L]$, so that $x$ encompasses the information of $S_{\mathrm{f}}^{\mathrm{mag}}[L]$ implicitly. This scheme is the same as what has been adopted in the lattice simulations of Ref. [9]. We compare these two methods for SU(3) color, and find qualitatively similar behavior.

\section{Results}

\subsection{SU(2)}

We first consider SU(2) color, to see how a mean-field approximation works when there is no sign problem. We look for a phase transition by considering how the Polyakov loop changes as $J$ increases. In the pure glue theory, the deconfining phase transition is known to be of second order for two colors, in the universality class of the Ising model. In our model, at $\varepsilon=0$ there is a 

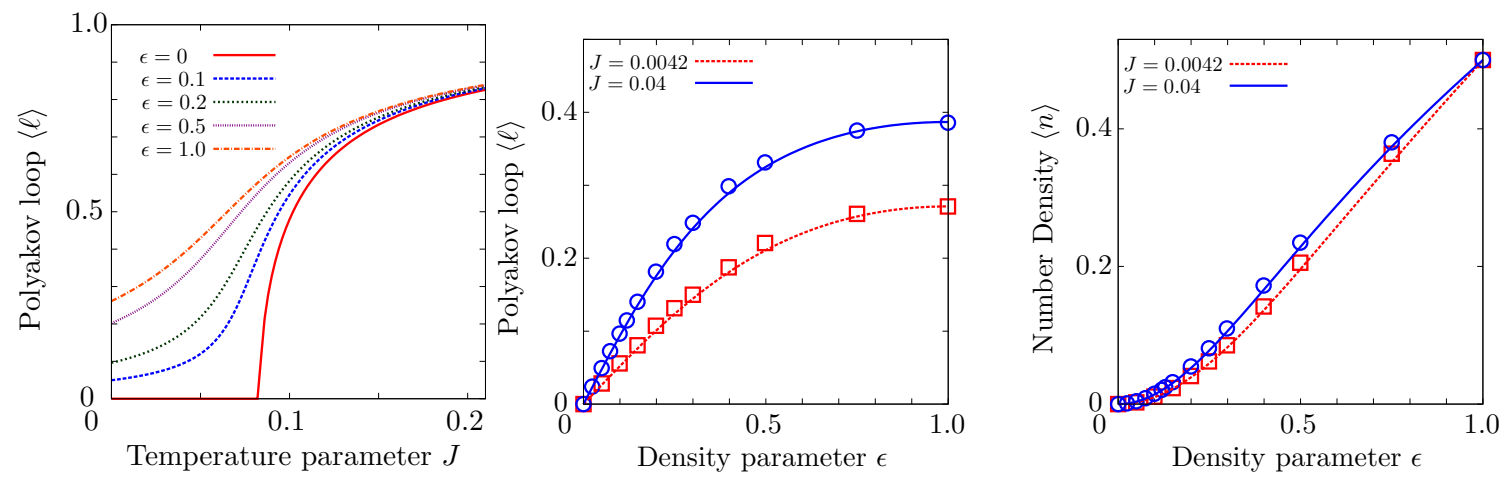

Figure 1: For SU(2), comparison of the model to lattice data, Fig. 2 of Ref. [9. Left: the Polyakov loop versus the temperature parameter. Center: the Polyakov loop versus the density parameter. Right: the number density versus the density parameter.

continuous transition at $J=J_{\mathrm{c}} \simeq 0.083$, as indicated by the solid curve in the left figure of Fig. 1, The presence of dynamical quarks acts on the Polyakov loop variable as an external field which breaks the center symmetry. In fact, the results at $\varepsilon \neq 0$ in the left figure of Fig. 1 indicate not a true phase transition, but only crossover.

Our mean-field outputs are to be compared with the lattice simulations in Ref. [9]: the center and left figures in Fig. 1 correspond to Figs. 1 and 2 of Ref. [9], respectively. We cannot expect exact agreement, because our ansatz for the pure gluonic action $S_{\mathrm{g}}[L]$ is only a crude approximation of QCD, and in any case, we neglect the renormalization of the Polyakov loop in a mean-field analysis. Nevertheless, the agreement turns out to be surprisingly good, beyond naive expectation, if the parameter $J$ is treated as an adjustable parameter as a fitting parameter. In this way, we fix $J=0.0042$ and $J=0.04$ to reproduce the SU(2) Polyakov loop only at $\varepsilon=1$ for $4 / g^{2}=2.0$ and $4 / g^{2}=1.5$, respectively. We stress that we do not use the data of the Polyakov loop at $\varepsilon \neq 1$, nor the results on the number density. Nevertheless, as clearly seen from Fig. 1, our numerical results fit all of the lattice data remarkably well. We conclude from this that the main corrections to our ansatz (2.3) can be represented by a shift in the parameter $J$. This gives us confidence in using a mean-field approximation for this problem.

\section{$4.2 \mathrm{SU}(3)$}

We next consider SU(3) color. The Polyakov loop is compared in the phase reweighting method, and the saddle point approximation, in the left figure in Fig. 2. this is to be compared with the lattice results from Fig. 7 of Ref. [9]. We find a first-order phase transition for $\varepsilon=0$ at $J=J_{\mathrm{c}}=0.132$ and for $\varepsilon=0.1$ at $J=J_{\mathrm{c}}=0.123$. Nonzero $\varepsilon$ smears the transition, so that it eventually ceases to be of first-order. The line of first-order transitions ends with a second order transition, which is then a critical end-point. For larger values of $\varepsilon$ there is only crossover. The global picture is consistent with results from a Potts model. In the left figure in Fig. 2 Jone sees that both reweighting, and the saddle-point approximation, have qualitatively the same behavior for the expectation value of the Polyakov loop.

At $\mu_{q} \neq 0,\langle\ell\rangle \neq\left\langle\ell^{*}\right\rangle$. The observable $\left\langle\ell-\ell^{*}\right\rangle$ is $C$-odd, where the imaginary part of the fermion determinant is responsible for this difference. In the center figure of Fig. 2 we present our 

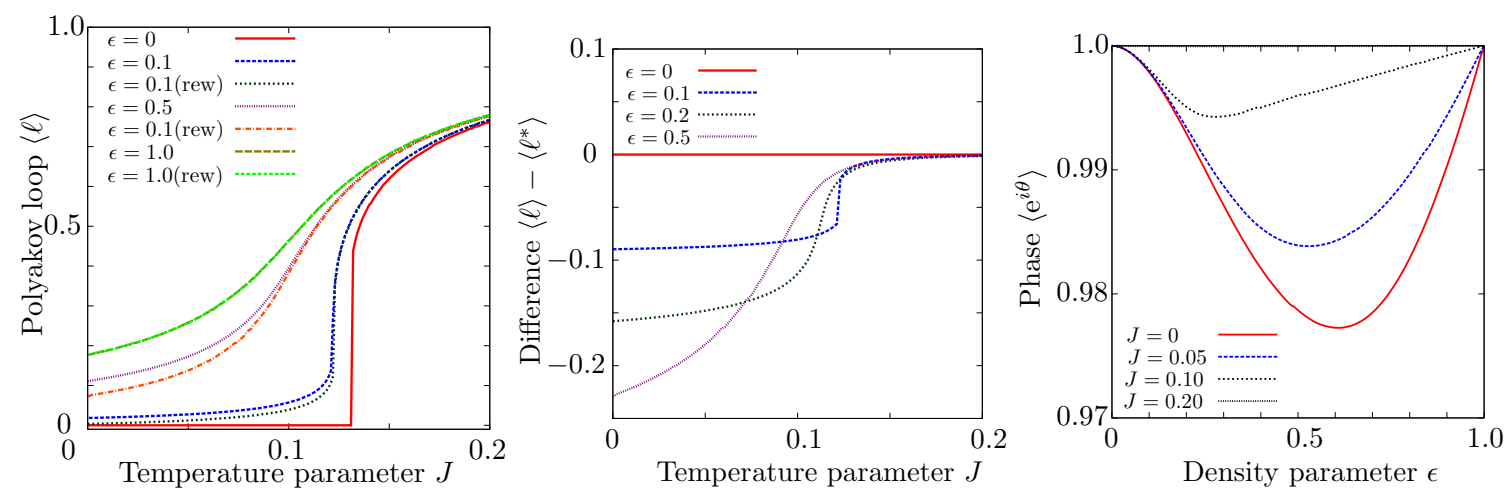

Figure 2: For SU(3), comparison of the model to lattice data, Fig. 2 of Ref. [9. Left: comparison of the Polyakov loop between phase reweighting and the saddle point approximation. Center: difference between $\langle\ell\rangle$ and $\left\langle\ell^{*}\right\rangle$, versus the temperature parameter. Right: expectation value of the phase of the quark determinant versus the density parameter.

numerical results for the difference $\langle\ell\rangle-\left\langle\ell^{*}\right\rangle$ as a function of $J$. The difference is trivially zero at $\varepsilon=0$ and $\varepsilon=1$ where the fermion determinant is real. As long as the density parameter stays smaller than $\varepsilon \sim 0.5$, a larger density parameter $\varepsilon$ leads to a bigger difference. For example, at $\varepsilon=0.5$ we find $\langle\ell\rangle-\left\langle\ell^{*}\right\rangle=-0.076$, which is comparable to $\langle\ell\rangle=0.073$.

One can intuitively understand why $\left\langle\ell^{*}\right\rangle$ is greater than $\langle\ell\rangle$ at nonzero $\mu_{q}$, as seen in [6]. It is because at nonzero quark density, the presence of quarks enhances the screening of antiquarks, so that an antiquark costs less energy [6].

Finally we present the results for the expectation value of the phase factor of the quark determinant, $e^{-i \Theta}$. We plot $\left\langle e^{-i \theta}\right\rangle$ as a function of $\varepsilon$ in Fig. 2, where $\theta$ is the phase at each lattice site; $\theta \equiv-\arg \left(1+\varepsilon^{3}+3 \varepsilon \ell+3 \varepsilon^{2} \ell^{*}\right)$, i.e. $\Theta=\sum_{\vec{x}} \theta$. Comparing it with Fig. 9 in Ref. [9], we see that our results qualitatively reproduce the lattice data. For more quantitative agreement, we approximate the phase factor by $\left\langle e^{-i \theta}\right\rangle^{216}$, taking the lattice volume of $6^{3}=216$ from [9]. For instance, our $J=0$ result has a minimum at $\varepsilon=0.61$ where $\left\langle e^{-i \theta}\right\rangle \simeq 0.977$, while we obtain $0.977^{216}=0.0066$. The minimum value in Fig. 9 of Ref. [9] is $\approx 0.01$, which is close to our value.

\section{Summary}

We have explored a simple model applicable in the limit of heavy quark mass and large chemical potential, and seen how the sign problem, at nonzero quark density, manifests itself in a meanfield approximation. All results from mean-field theory are reasonable, and are in quantitative agreement with lattice data. There is no sign problem for $\mathrm{SU}(2)$, and we find that a mean-field approximation works well, for both the quark number density and the Polyakov loop.

For SU(3), we compared two methods, a saddle point approximation with a complex action, and phase reweighting. We find that both methods give qualitatively the same behavior for the expectation value of the Polyakov loop. The complex action implies that $\langle\ell\rangle \neq\left\langle\ell^{*}\right\rangle$, which is also seen with phase reweighting. We computed $\langle\ell\rangle-\left\langle\ell^{*}\right\rangle$, as a function of the parameters for temperature and density. 
While it may appear odd to find a saddle point in the mean field approximation, it is known that this happens in other theories, such as for a nonlinear sigma model. There, the constraint of the nonlinear model is eliminated by introducing a new field. The effective action, including the constraint field, is complex, so that the stationary points thereof are true saddle points. For QCD, the $A_{0}$ field is a constraint field which imposes Gauss' law. This may provide a clue to resolving the sign problem with dense quarks.

\section{References}

[1] K. Fukushima and Y. Hidaka, Phys. Rev. D 75, 036002 (2007) [arXiv:hep-ph/0610323].

[2] M. Cheng et al., Phys. Rev. D 74, 054507 (2006) [arXiv:hep-lat/0608013]; Y. Aoki, Z. Fodor, S. D. Katz and K. K. Szabo, Phys. Lett. B 643, 46 (2006) [arXiv:hep-lat/0609068].

[3] Y. Aoki, Z. Fodor, S. D. Katz and K. K. Szabo, towards JHEP 0601, 089 (2006) [arXiv:hep-lat/0510084].

[4] C. Bernard et al. [MILC Collaboration], Phys. Rev. D 71, 034504 (2005) [arXiv:hep-lat/0405029].

[5] Z. Fodor and S. D. Katz, Phys. Lett. B 534, 87 (2002) [arXiv:hep-lat/0104001]; mu,” JHEP 0203, 014 (2002) [arXiv:hep-lat/0106002]; JHEP 0404, 050 (2004) [arXiv:hep-lat/0402006].

[6] C. R. Allton et al., Phys. Rev. D 66, 074507 (2002) [arXiv:hep-lat/0204010]; C. R. Allton, S. Ejiri, S. J. Hands, O. Kaczmarek, F. Karsch, E. Laermann and C. Schmidt, potential,” Phys. Rev. D 68, 014507 (2003) [arXiv:hep-lat/0305007].

[7] M. G. Alford, A. Kapustin and F. Wilczek, lattice," Phys. Rev. D 59, 054502 (1999) [arXiv:hep-lat/9807039]; P. de Forcrand and O. Philipsen, Nucl. Phys. B 642, 290 (2002) [arXiv:hep-lat/0205016]; Nucl. Phys. B 673, 170 (2003) [arXiv:hep-lat/0307020]; [arXiv:hep-lat/0607017]; M. D’Elia and M. P. Lombardo, Phys. Rev. D 67, 014505 (2003) [arXiv:hep-lat/0209146].

[8] A. Dumitru, R. D. Pisarski and D. Zschiesche, Phys. Rev. D 72, 065008 (2005) [arXiv:hep-ph/0505256].

[9] T. C. Blum, J. E. Hetrick and D. Toussaint, Phys. Rev. Lett. 76, 1019 (1996) The published version of this paper does not contain the results for SU(2), which are in [arXiv:hep-lat/9509002]; we refer only to the later. 